\title{
Data Mining Based on Compensation Fuzzy Neural Networks
}

\author{
Ze Min Qiu * \\ Xinhua College of Sun Yat-sen University, Guangzhou 510520, China \\ 254434193@qq.com
}

Keywords: Neural networks; Data mining; Compensate; Training; Convergence.

\begin{abstract}
The purpose: Solve the existing problems of fuzzy neural network are processing speed and low accuracy. Methods: This paper presents a data mining based on compensation fuzzy neural networks. Through research and analysis of the traditional compensation neural network, we are optimized it according to the characteristics of data mining. And optimize the calculation and training of the data mining. The result: In the first group can be seen that using this algorithm can be get a higher convergence. In the case of less data, the accuracy of the proposed algorithm is almost the same with literature algorithm. In the case of a large amount of data, the proposed method is better than other algorithms. In conclusion: The experimental results basically consistent with the expected results. It can maintain convergence effect, while ensuring the accuracy of the network.
\end{abstract}

\section{Introduction}

With the development and popularization of technologies, data mining are also constantly developing. It is widely used in the field of artificial intelligence and information processing. And now, Data mining technology is from the laboratory into the business. In particular, many enterprise-level companies have themselves data warehouse resources. Data mining has become one of the important research topics. In other countries, they have powerful data mining tools like the Nuggets. In China, this research is still at the stage of theoretical study and discussion. Nowadays, researchers were put forward a number data mining algorithms. For example, Data mining algorithm based on fuzzy sets [1-2], data mining algorithm based on clustering algorithm [3-4], Data mining algorithms based on neural networks[5-6], etc. However, we can found that these algorithms still have lot problems in practical. Although these can extract the valid information in the large data, but the algorithm has a great constraint because of the complexity and the noise. Further development of artificial intelligence technology and computer technology, the compensative Fuzzy Neural Network get promoted. It is a hybrid system combines with the compensation fuzzy logic and neural networks. To introduce the compensation fuzzy neurons, this can make right from the initial definition of fuzzy rules. It can make the network more fault tolerance and the system become more stable.

Therefore, this paper studies Compensative Fuzzy Neural Network. Combined with its global optimization features, this paper presents a data mining based on compensation fuzzy neural networks. Through improved Compensative Fuzzy Neural Network, to make it get the better convergence. And through the establishment of the error function, optimized computing and training in the systems. So that it can maintain good accuracy. 


\section{Fuzzy Neural Network}

System Architecture. Fuzzy neural networks have different ways of combining fuzzy logic and neural network [8]. The first, it is still using ordinary neural network structure, but using fuzzy arithmetic neurons instead of ordinary nonlinear neurons. The second, it is using ordinary neural network structure and neurons as information processing tools. The structure of Fuzzy neural network system is shown in Figure 1[9].

\section{Fuzzification $\rightarrow$ Fuzzy Inference of the Neural Networks $\rightarrow$ Clarity and feedback correction}

Figure. 1 System architecture of the fuzzy neural network

Compensation fuzzy neural network is divided into five levels in the paper. These are input layer, fuzzy layer, fuzzy inference layer, operation layer and anti-blur compensation layer. The following detailed analysis it. In the first layer, each node is directly connected with the input vector. In the second layer, each node represents a linguistic variable value. It is can calculate the membership degree of every branch of the input vector belonging to the corresponding fuzzy set of each linguistic variable. In the third layer, the neural node represents a fuzzy rule. They can match the fuzzy rules and calculate the fitness degree of each rule. In the fourth layer, neurons carry out the compensative fuzzy computations. And in the fifth layer, neurons carry out the anti-fuzzy computations to obtain the exact values of the network's outputs. Between layers build was based on fuzzy logic system linguistic variables, fuzzy rules, the worst one the best computing, fuzzy reasoning method, the anti-blur function. Not only can properly adjust the input and output fuzzy membership function, but also by means of compensation logic optimization algorithm to dynamically adapt the fuzzy inference. That has a clear physical meaning.

\section{Article algorithm}

Improved compensation fuzzy neural network. In the training process, iteration may be divided into two processes [10]: the forward compensative fuzzy reasoning process and the backward error propagation process. At the beginning of training, initial values should be preset for the network's parameters including the center and width of fuzzy membership functions for input and output, together with the compensation degree. Then the compensative fuzzy reasoning and error's counter spreading process will be iterated until the result is satisfying. Method is as follows.

Set up a network with $\mathrm{n}$ input, m output, $\mathrm{S}$ fuzzy inference rules. $\mathrm{P}$ sample signal $\left(x^{p}, y^{p}\right)$ using training network. Fuzzy membership function of input and output are Gaussian as follows.

$$
\mu_{A_{i}^{k}}\left(X_{i}\right)=\exp \left[-\left(\frac{X_{i}-a_{i}^{k}}{\sigma_{i}^{k}}\right)^{2}\right] ; \mu_{B_{j}^{k}}\left(y_{j}\right)=\exp \left[-\left(\frac{y_{j}-b_{j}^{k}}{\omega_{j}^{k}}\right)^{2}\right]
$$

Therein $a_{i}^{k}$ representative of the center, $\sigma_{i}^{k}$ representative of the width of the membership function of input, $b_{j}^{k}$ representative of the center, $\omega_{j}^{k}$ representative of the width of the membership function of the output.

In practice, the sum of the widths of the input and output variables usually equal to the value of the variable. Center and width of the membership function often depends on the distribution of variables. The accuracy and time to train directly affects the number of nodes in the hidden layer. Therefore, these are two factors into account in the paper.

The fuzzy reasoning rule $\mathrm{k}$ among the $\mathrm{R}$ ones is described as follows: 


$$
R_{k}: \text { If }\left(\mathrm{x}_{1}=A_{i}^{k} \text { and } \mathrm{x}_{2}=\mathrm{A}_{2}^{\mathrm{k}} \text { and } \cdots \text { and } \mathrm{x}_{\mathrm{n}}=\mathrm{A}_{\mathrm{n}}^{\mathrm{k}}\right)
$$

Then $\left(\mathrm{y}_{1}=\mathrm{B}_{1}^{\mathrm{k}}\right.$ and $\mathrm{y}_{2}=\mathrm{B}_{2}^{\mathrm{k}}$ and $\cdots$ and $\left.\mathrm{y}_{\mathrm{n}}=\mathrm{B}_{\mathrm{n}}^{\mathrm{k}}\right)$

fuzzy set $\mathrm{A}$ within the range of $U=U_{1} \times U_{2} \times \cdots U_{n}$, Output fuzzy set $\mathrm{B}$ in the range of $V=V_{1} \times V_{2} \times \cdots V_{n}$; Then it would be transformed by the fuzzy reasoning rule $\mathrm{k}$ in the third layer.

After reasoning operation, operation degree of membership: $\mu_{B} k=\left[\prod_{i=1}^{n} \mu_{A_{i}} k\left(x_{i}\right)\right]^{1-r_{k}+r_{k} / n}$

The degree of compensation $r_{k} \in[0,1]$

Output value is the fifth layer obtained after normalization sub-operations.

$$
\tilde{y}_{j}=\frac{\sum_{k=1}^{k} b_{j}^{k} \omega_{j}^{k} Z_{k}}{\sum_{k=1}^{s} \omega_{j}^{k} Z_{k}} \text {. Therein } z_{k}=\left[\prod_{i=1}^{n} \mu_{A_{i}} k\left(X_{i}\right)\right]^{1-r_{k}+r_{k} / n}
$$

The objective function: $E=\frac{1}{2} \sum_{i=1}^{m}\left(\tilde{y}_{j}^{p}-y_{j}^{p}\right)^{2}$

The gradient descent law which can adjust to train the compensation degree in the compensative computing, the center and width of the input / output membership functions. L.e.

$$
\begin{aligned}
& b_{j}^{k}(t+1)=b_{j}^{k}(t)-\eta \frac{\partial E}{\partial b_{j}^{k}(t)} ; \quad \omega_{j}^{k}(t+1)=\omega_{j}^{k}(t)-\eta \frac{\partial E}{\partial \omega_{j}^{k}(t)} \\
& a_{j}^{k}(t+1)=a_{j}^{k}(t)-\eta \frac{\partial E}{\partial a_{j}^{k}(t)} ; \quad \sigma_{j}^{k}(t+1)=\sigma_{j}^{k}(t)-\eta \frac{\partial E}{\partial \sigma_{j}^{k}(t)}
\end{aligned}
$$

Degree of compensation $r_{k} \in[0,1]$, therein, Set up $r_{k}=\frac{c_{k}^{2}}{c_{k}^{2}+d_{k}^{2}}$

Satisfy the following relationship formula:

$$
\frac{\partial E}{\partial c_{k}}=\frac{\partial E \partial r_{k}}{\partial r_{k} \partial c_{k}}=\frac{2 c_{k} d_{k}^{2} \partial E}{\left(c_{k}^{2}+d_{k}^{2}\right)^{2} \partial r_{k}} ; \frac{\partial E}{\partial d_{k}}=\frac{\partial E \partial r_{k}}{\partial r_{k} \partial d_{k}}=\frac{-2 c_{k}^{2} d_{k} \partial E}{\left(c_{k}^{2}+d_{k}^{2}\right)^{2} \partial r_{k}}
$$

Therein, $\eta(t)$ represents a learning efficiency, $t$ representative of the training step.

At the end, convergence judge with this algorithm. When objective function satisfies the equation $E(t)<\varepsilon$, The network stop learning and output final results. $\varepsilon$ is a small positive number. Otherwise, let $\mathrm{t}+1$, and iteration continues to run.

Optimization calculation and training. In practical application, we can see the data in the network is easy to interference by external factors. It must lead some deviation from the actual value of the original value. Therefore, In order to make the algorithm more reliable information, this paper is introducing an error function F. Error function expression is as \[ F=-\sum_{i=1}^{k} \mu_{A_{i}^{k}}\left(X_{i}\right) \ln A_{A_{i}^{k}}\left(X_{i}\right)+\left(1-\mu_{A_{i}^{k}}\left(x_{i}\right)\right) \ln \left(1-A_{A_{i}^{k}}\left(X_{i}\right)\right) \]
follows.

$$
\text { Therein } \mu_{A_{i}^{k}}\left(X_{\dot{i}}\right) \text { is a fuzzy expected input value, }{ }_{A_{i}^{k}}^{A_{i}\left(X_{\dot{i}}\right)} \text { is the actual input values for the fuzzy. }
$$


In order for the error function can be applied to the crop of the network, we make network weights quickly become 0 . In training, this paper was combine with the error function and penalty function $p$.Function expression: $\quad p=\frac{X}{2}\left(\sum_{m=1}^{H} \sum_{l=1}^{N} W_{m l}^{2}+\sum_{m=1}^{H} \sum_{p=1}^{c} V_{p m}^{2}\right)$

Therein, ${ }^{W_{m}}$ are expressed vague connection weights between the input layer 1-th node and the hidden layer $\mathrm{m}$-th node. ${ }^{V_{p m}}$ are expressed vague connection weights between hidden layer m-th node and fuzzy output layer $\mathrm{p}$-th node. $\mathrm{H}$ is the total number of nodes in the hidden layer. $\mathrm{N}, \mathrm{C}$ is the total number of input and output. $X$ is the penalty function parameters, and $X$ determining a symmetrical interval centered 0. L.e :

$$
F=-p \sum_{i=1}^{k} \mu_{A_{i}^{k}}\left(X_{i}\right) \ln A_{A_{i}^{k}}\left(X_{i}\right)+\left(1-\mu_{A_{i}^{k}}\left(X_{i}\right)\right) \ln \left(1-A_{A_{i}^{k}}\left(X_{i}\right)\right)
$$

\section{Simulation}

Operational network, the network 100000 data are as experimental data in this paper. Simulation experiments on them. In order to verify the feasibility of the algorithm, Such as data mining algorithm base on traditional fuzzy neural network, and Literature algorithm [10]. The following graph, the circles represent the traditional algorithms, quadrilateral representative Literature algorithm, triangles represent article algorithm.

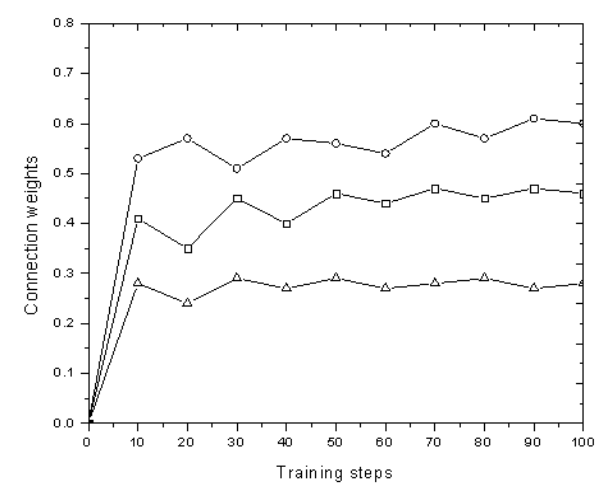

Figure. 2 Curve of training steps and connection weights

By observing Figure 2 we can see that the connection weights of each algorithm will eventually stabilized. The curves of article algorithm remain at the bottom of the other algorithms. It proved effective algorithm has better convergence. The number of steps in the training 20, this algorithm can remain stable. It was early in the literature algorithm and the traditional algorithm about 40-step. To further proof the convergence speed in article algorithm.

In order to compare the accuracy of convergence, this paper was choice of two different data to comparing. And this papers only the selected literature algorithm to compare in order to simplify the experiment. The simulation results are shown below. 


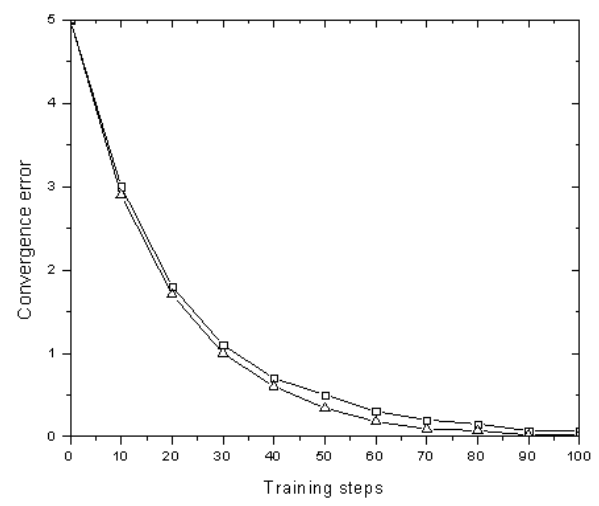

Figure. 3 The convergence error curve in case of less the amount of data

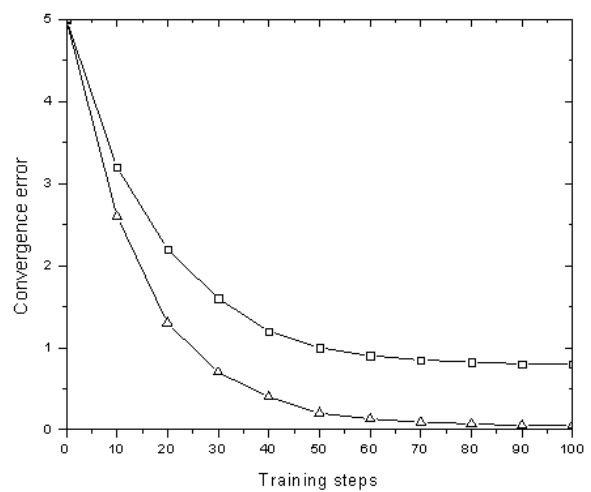

Figure. 4 The convergence error curve in case of large amounts of data

By observing Figures 3 and 4 can be seen that convergence error curve becomes smaller with increasing the number of training steps. In the last, it was maintained stable at a low value. When the network data is less, the convergence error value of this algorithm is almost the same the literature algorithm. This algorithm is slightly better than the literature algorithm. When the network is large amount of data, these algorithms is significantly better literature algorithm, and remain stable after $50 \mathrm{~s}$. Valid proof that the stability of the convergence precision.

\section{Summary}

Proposed data mining based on compensation fuzzy neural network. Base on compensatory fuzzy algorithm has characteristics of good global and dynamic, this paper is establishing the error function for optimization algorithm. And optimize the calculate and training process to provide data relevant to the efficiency and quality. Experimental results show that experimental results of this algorithm basically consistent with the expected results. Algorithm under different amounts of data, it can maintaining good convergence and stability. 


\section{References}

[1]Chen CY, Liang GS, Su Y, Liao MS. A data mining algorithm for fuzzy transaction data[J]. QUALITY \& QUANTITY. 2014,48(6): 2963-2971.

[2]Casillas J, Martinez-Lopez FJ. Mining uncertain data with multiobjective genetic fuzzy systems to be applied in consumer behaviour modelling $[\mathrm{J}]$. EXPERT SYSTEMS WITH APPLICATIONS. 2009,36(2): 1645-1659.

[3]Wang ZH, Tu L, Guo Z, Yang LT, Huang BX. Analysis of user behaviors by mining large network data sets[J]. FUTURE GENERATION COMPUTER SYSTEMS-THE INTERNATIONAL JOURNAL OF GRID COMPUTING AND ESCIENCE. 2014,37: 429-437.

[4]Farvaresh H, Sepehri MM. A data mining framework for detecting subscription fraud in telecommunication[J]. ENGINEERING APPLICATIONS OF ARTIFICIAL INTELLIGENCE. 2011,24(1): 182-194.

[5]Simeunovic V, Preradovic L. Using Data Mining to Predict Success in Studying[J].Croatian journal of education-hrvatski casopis za odgoj I obrazovanje. .2014,16(2): 491-523.

[6]Mostafa MM, El-Masry AA. Citizens as consumers: Profiling e-government services' users in Egypt via data mining techniques[J].INTERNATIONAL JOURNAL OF INFORMATION MANAGEMENT. 2013,33(4): 627-641.

[7] Keller BSB, Kim JS, Steiner PM. Abstract: Data Mining Alternatives to Logistic Regression for Propensity Score Estimation: Neural Networks and Support Vector Machines[J]. MULTIVARIATE BEHAVIORAL RESEARCH. 2013,48(1): 164-164.

[8]Liu WG, Seto KC. Using the ART-MMAP neural network to model and predict urban growth: a spatiotemporal data mining approach[J]. ENVIRONMENT AND PLANNING B-PLANNING \& DESIGN. 2008,35(2): 296-317.

[9]Chen CY, Chiang JS, Chen KY, Liu TK, Wong CC. An Approach for Fuzzy Modeling based on Self-Organizing Feature Maps Neural Network[J]. APPLIED MATHEMATICS \& INFORMATION SCIENCES. 2014,8(3):1207-1215.

[10]Haeri A, Tavakkoli-Moghaddam R. DEVELOPING A HYBRID DATA MINING APPROACH BASED ON MULTI-OBJECTIVE PARTICLE SWARM OPTIMIZATION FOR SOLVING A TRAVELING SALESMAN PROBLEM[J]. JOURNAL OF BUSINESS ECONOMICS AND MANAGEMENT. 2012,13(5): 951-967. 